\title{
IMAGINARIOS ASTRONÓMICOS EN EL PERÚ: SIGLOS XVII-XIX
}

\author{
Roberto Juan Katayama Omura \\ Universidad Ricardo Palma
}

\section{RESUMEN}

En el presente artículo mostramos los imaginarios astronómicos en el Perú de los siglos xvii, xviii y xix. Se determinarán que fueron el de Tycho Brahe, Ptolomeo y Kepler-Newton. La adopción de estos imaginarios estuvo relacionada con la adopción de la física moderna y una ontología mecanicista.

\section{PALABRAS CLAVE}

Sistema ticónico / Sistema ptolemaico / Sistema / Kepleriano-newtoniano / Ontología.

\begin{abstract}
In this article we show the astronomical imaginaries in Perú in xvii, xviii and xix centuries. We will determine that these were Tycho Brahe's system, Ptolomeus's system and Kepler-Newton's system. The adoption of these imaginaries was in relation with the adopction of modern physics and a mechanic ontology.

KEY WORDS

Ticonic's system / ptolomeus's system / System / Kepler-Newton system / Ontology.
\end{abstract}




\section{EL IMAGINARIO CLÁSICO}

\section{EL SISTEMA DE PTOLOMEO}

Una vez que los españoles arribaron a las costas americanas e iniciaron la conquista del continente, la navegación de altura cobró un papel relevante no sólo por temas de exploración y control territorial sino también por temas de navegación ultramarina, seguridad, dominio de los mares e interconexión entre la América española y la península ibérica. Dentro de este contexto las cartas de navegación y el estudio del cielo jugaron un papel preponderante en una época donde las estrellas eran el único punto de orientación en los mares y en donde la vida de los pasajeros y el desarrollo del comercio ultramarino dependían de la habilidad de los pilotos de las naos.

Como ha sido señalado por historiadores españoles especialistas en el tema de la historia de la navegación, el sistema del mundo supuesto en las cartas de navegación elaboradas durante el siglo xvi y gran parte del siglo xvii suponen el sistema geocéntrico de Claudio Ptolomeo:

Las bases teóricas de la astronomía práctica de la España renacentista, tenían su origen en la cosmología tradicional ... los textos publicados en aquellos años eran normalmente una revisión del sistema de ptolomaico presentado en el Tractatu de Sphera, escrito en el siglo XIII por Joannes de Sacrobosio. (Gonzáles, 1992, p. 26)

Incluso en el siglo xviii como Joseph Eusebio de Llano y Zapata se nos mostrará como partidario del sistema de Ptolomeo. Como la demanda de pilotos seguía aumentando, los graduados en España no lograban satisfacerla, por ello, durante el gobierno del Príncipe de Esquilache (1615-1621) en el virreinato del Perú se crea el cargo de "Cosmógrafo Mayor del Reino".

En el siglo XVII cinco fueron los que ostentaron el cargo de Cosmógrafo Mayor del Reino: Lucas de Quirós (1618-1634), Francisco de Quirós (1619-1645), Diego de León (1645-1661), Francisco Ruiz Lozano (16621677) y Juan Ramón Connig (1678-1708). En el siglo XVII cinco fueron los que ostentaron el cargo de Cosmógrafo Mayor del Reino: Lucas de Quirós (1618-1634), Francisco de Quirós (1619-1645), Diego de León (1645-1661), Francisco Ruiz Lozano (1662-1677) y Juan Ramón Connig (1678-1708).

Federico Schwab en su célebre artículo "Los Almanaques Peruanos" nos informa que durante el resto de periodo virreinal se sucedieron en el cosmografiato, Pedro de Peralta Barnuevo (1709-1743), José de Mosquera y 
Villaroel (1744-1749), Juan Rer (1750-1756), Cosme Bueno (1757-1798), Gabriel Moreno (1799-1809), José Gregorio Paredes (1810), Francisco Romero (1811-1813) y nuevamente José Gregorio Paredes (1814-1825). (Schwab, 1948).

\section{EL SISTEMA DE BRAHE}

Como hemos demostrado en anteriores investigaciones sobre el tema por lo menos desde Ruiz Lozano hasta Juan Rer todos los cosmógrafos se muestran inmersos dentro del paradigma clásico, en su versión ticónica. Tomemos, a modo de ejemplo, el caso de Ruiz Lozano.

El catedrático sanmarquino expone sus ideas en un texto titulado "Tratado de cometas"1, publicado el año de 1665 y escrito con motivo de la aparición de uno de estos astros entre fines de 1664 y que se mantuvo en los cielos hasta inicios de 1665 .

En cuanto al sistema del mundo que seguiría Ruiz Lozano, hay dos pasajes decisivos que nos han permitido establecerlo textualmente. En el primero de ellos Ruiz sostiene que los planetas describen órbitas que los alejan o acercan del centro de la Tierra:

Sea la primera mudança de los Abfides de los Planetas, que fe mudan fegun la fuceffion de Signos, mediante la velocidad, o tardança del movimiento, haziendo tranfitos de unos lugares en otros; donde vnas vezes eftan altiffimos y apartados del centro de la Tierra, y otras muy cercanos a ellas en diferentes Signos de Zodiaco. (Lozano, 1665, p. 14).

En el segundo se sostiene que todos los planetas (no menciona a la Tierra, por lo que se puede inferir que no la considera "planeta") están ligados al Sol y se mueven respecto a éste:

... la mudança de la eccentricidad del Sol, a quien no fola la Venus,

y Mercurio, cuyos medios movimientos fon los mifmos que el del

1 El Tratado de cometas, observacion, y ivivio del que se vio en esta civdad de los Reyes, y generalmente en todo el Mundo, por los fines del año de 1664. Y principios defte de 1665. Compuesto por el Capitan Francisco Rviz Lozano Cofmografo mayor defte Reyno, y Cathedratico de Prima de Mathematicas en efta Ciudad, tal el título completo de la obra, fue publicado por Francisco Ruiz Lozano el año de 1665 en la ciudad de Lima y consta de 42 hojas dobles de texto efectivo. 
Sol, sino los demas Planetas Saturno, Iupiter, Marte, y Luna muda todos al mismo punto fus eccentricidades; porque en buena Theorica de Orbes eftan ligados al Sol de fuerte que a fu refpecto folo se mueuen. (Lozano, 1665, p. 16).

Dentro del paradigma geocéntrico ambos fenómenos pueden darse únicamente en el sistema de Tycho Brahe, en éste los cinco planetas conocidos (Mercurio, Venus, Marte, Júpiter y Saturno) giran alrededor del Sol pero éste gira alrededor de la Tierra. En ese sentido las órbitas descritas por los planetas los acercan o alejan del centro de la Tierra al tiempo que se mueven alrededor del sol.

\section{EL IMAGINARIO MODERNO: EL SISTEMA DE KEPLER}

Es el imaginario moderno propiamente dicho. Por "astronomía moderna" entendemos aquella que surgió en Europa entre los siglos xvi y xvii y que posibilitó el paso de un modelo geocéntrico a otro heliocéntrico. Uno de sus hitos iniciales fue la llamada "revolución copernicana" y su culminación la "Teoría de la Gravitación Universal" de Newton. Junto con ella se instaura una ontología física cuantitativa y una gnoseología causal que es a la vez lineal y mecanicista, como lo sostendrá Galileo:

La filosofía está escrita en ese grandísimo libro que tenemos abierto ante los ojos, quiero decir, el universo, pero no se puede entender si antes no se aprende a entender la lengua, a conocer los caracteres en los que está escrito. Está escrito en lengua matemática y sus caracteres son triángulos, círculo y otras figuras geométricas, sin las cuales es imposible entender ni una palabra; sin ellos es como girar vanamente en un oscuro laberinto. (Galileo, 1984, p. 61).

La figura en cuya época se iniciaría el lento paso del paradigma clásico al moderno -sin que por ello sea él mismo propia y totalmente moderno- sería Cosme Bueno, quien sumió el cosmografiato el año de 1757 y se mantuvo en éste hasta 1798. Con él parecería que comenzaría el lento cambio en el sistema del mundo imperante en el pensamiento astronómico virreinal peruano. Pues al parecer tendría cierta influencia de Newton, lo que se puede ver tanto en algunos de sus propios textos como mediante el testimonio de sus discípulos. Así, por ejemplo, Bueno se muestra de acuerdo con las tesis del newtoniano David Gregori, en relación a los efectos negativos que podrían tener los cometas sobre la Tierra:

Si estos vapores, añade David Gregori celebre Newtoniano, difundido por aquellos vaftos efpacios caen hacia nofotros, podrán alterar 
el Ayre ... caufar mucho daño al Reyno vegetal, y Animal... (Bueno, 1756$)^{2}$

Por su parte, Gabriel Moreno, discípulo de Cosme Bueno y sucesor suyo en el cosmografiato, sostendrá la influencia de la Teoría de la Gravitación Universal newtoniana en el pensamiento de su maestro, cual comenzó a tomar fuerza en el virreinato peruano a raíz de la llegada de la misión científica francesa de Charles de la Condamine, a mediados del siglo xviii:

Hablando del físico de Newton, decía que los Académicos de Paris enviados al Perú, fueron Apóstoles del Filósofo Inglés, iniciados en lo que debían predicar en sus montañas á favor de la atracción. (Moreno, 1798)

Por otro lado, el mismo Moreno sostiene que Cosme Bueno fue el primer astrónomo de tendencia newtoniana en el virreinato peruano: "Primer prosélito de Neuton en el Perú, adquirió la regla y exactitud de su espíritu á fuerza de estudiarlo" (Moreno, 1798).

Sin embargo, a pesar de estas referencias de sus discípulos, la evidencia textual hallada por nosotros en los textos del propio Cosme Bueno no es tan contundente, pues en algunos pasajes de sus propios textos parecería discrepar de los newtonianos, así, por ejemplo, en el ejemplar El Conocimiento de los tiempos correspondiente al año de 1758 cita a un famoso astrónomo newtoniano al tiempo que expresa sus dudas sobre los cálculos de las órbitas de los cometas efectuados bajo el sistema de Newton:

Tres especialmente fon los Cometas cuyo retorno fe predice mas ha de 50 años; que fueron el que apareció el año de 1531. el del año de 1660. y el de 1680. El primero, fegun el computo de los Neutonianos, debe volverfe a ver en el prefente de 1758 ... No falgo por fiador de fus cálculos, por motivos de larga difcufsion. (Bueno, 1757)

En ese mismo ejemplar, en un pasaje en el cual discute cómo puede ser que un cometa ejerza un influjo negativo en la Tierra, sostiene que ello puede darse sobre todo si el cometa aparece entre el Sol y la Tierra, ya que entonces, estaría más cercano a nosotros:

De todo lo hafta aquí brevemente dicho se colige que fi cuando fe verifica la predicción, podremos temer en los tiempos fubfequentes

2 Los Almanaques se imprimían y publicaban a fines del año anterior sobre el que trataban, por tema de distribución. De ahí que el año que se refiere es el año de publicación, no el año del que se ocupa el Almanaque. 
algunas de aquellas alteraciones que correfponden á fus malignos influxos: principalmente fi aparece entre el Sol y la tierra; pues como mas vecino entonces á nosotros ... (Bueno, 1757).

Esto no es posible en el sistema de Ptolomeo ya que entre el Sol y la Tierra están las órbitas de la Luna, Mercurio y Venus respectivamente. Tampoco en posible en el sistema heliocéntrico pues en éste, entre el Sol y la Tierra están las órbitas de Mercurio y Venus. Sin embargo, el que un cometa aparezca entre el Sol y la Tierra y que ello implique que dicho cometa está más cercano a nosotros que a los otros planetas sí es posible en el sistema de Tycho Brahe debido a que en éste, la Tierra ocupa el centro del cosmos y alrededor de ella primero orbita el Sol, estando los demás planetas orbitando alrededor del Sol. Por ello, en este sistema, si un cometa aparece entre el Sol y nosotros, obviamente está muy cercano a la Tierra.

Por lo anterior, Cosme Bueno se nos muestra más bien como partidario del geocentrismo ticónico. Así, parece que en el caso de Bueno habría un marcado eclecticismo; parecería que en algunos aspectos aceptara las modernas tesis de los newtonianos pero en otros, como el sistema del mundo, seguiría siendo seguidor de Brahe. Como señalara su propio discípulo, Gabriel Moreno; "D. Cosme en su vejez ya se reía de todos los sistema" (Moreno, 1798)

Respecto a este punto podríamos argumentar que una lectura heterodoxa de los Principia de Newton, podría hallar fundamento para este eclecticismo cosmológico, por ejemplo, en el Libro III, en el capítulo titulado "Fenónenos", Newton escribe:

Supuestas en reposo las estrellas fijas, los tiempos periódicos de los cinco planetas primarios y del Sol en torno a la Tierra o de la Tierra en torno al Sol están en razón de la potencia 3/2 de las distancias medias al Sol. (Newton, 2011, p. 622).

En seguida Newton explica lo anterior con la siguiente frase: "Esta razón hallada por Kepler está reconocida por todos. Pues, los tiempos periódicos son los mismos y las magnitudes de las órbitas las mismas tanto si el Sol gira en torno a la Tierra como si la Tierra gira en torno al Sol" (Newton, 2011, p. 623).

De ahí podría inferirse, para motivos de cálculo puro, aparentemente tanto la corrección de la posición kepleriana como la braheana. 
En ese sentido, parecería que Cosme Bueno seguía a Newton en algunos aspectos pero en otros, principalmente el relativo a la ontología cósmica, parecería que estaba aún dentro de la tradición clásica.

El sucesor de Cosme Bueno en el cosmografiato fue su discípulo Gabriel Moreno, fue cosmógrafo entre 1799 y 1809. Es definitivamente seguidor de Newton. Así, en el ejemplar de Almanaque peruano y guía de forasteros publicado a fines de 1799 (en este año se produce el cambio de nombre de El conocimiento de los tiempos..., al de Almanaque peruano... que estamos indicando más atrás) y correspondiente al año de 1800 sostendrá que respecto a la astronomía, en los dos últimos siglos se ha llevado a cabo una revolución que ha culminado con la gravitación universal de Newton:

En los dos últimos siglos, en que han sido tan rápidos sus progresos, debidos á la exactitud de las observaciones y aplicación de las fuerzas de Neuton á las leyes de Keplero (Moreno, 1798).

La relevancia del sistema kepleriano-newtoniano, por otro lado, se debe, en palabras de Moreno a "que hoy rige en la Astronomía, explicando felizmente todos los phenómenos celestes" (Moreno, 1798)

Esto lleva a que Moreno, en el ejemplar para el año de 1806, (publicado el año de 1805), a reconocer los aportes de Descartes, Kepler, Leibnitz y Newton al desarrollo de las matemáticas y la astronomía:

Descartes en la Francia, Keplero, Leibnitz en Alemania variaron el aspecto de las ciencias Exactas. La Algebra que desde su origen había dado algunos pasos adquirió baxo de la mano del primero, sino toda la perfeccion, la limpieza de sus expresiones y la aplicación a un Geometra. La Astronomia recibió del segundo las dos admirables Leyes que reynan en el movimiento de los Astros y la figura de las órbitas que corren. La Geometría debe al tercero la luz y facilidad con que el calculo diferencial penetra todas sus partes, principalmente el país inagotable de las curvas, y sus propiedades. Estos progresos guiadores de los Newton, Benouli y Euler... (Moreno, 1805).

En la senda propiamente moderna atisbada por Bueno y desarrollada integralmente por Moreno se mantendrá a partir de ahora la élite científicoastronómica peruana.

Este imaginario moderno se puso en evidencia en la prensa peruana a raíz de un cometa sobre cuyo paralaje se suscitó una pequeña polémica. 
Así, durante el mes de marzo del año de 1843 se publicó un supuesto estudio sobre la órbita de un cometa que se observó por esas fechas. Al año siguiente el Capitán de Navío Eduardo Carrasco (en ese entonces Cosmógrafo de la República) lanzó una furibunda crítica:

Como éstas circunstancias asertivas son deducidas de una órbita imajinaria, no es de extrañar se hayan falsificado, según inferimos debían suceder, pues el Cometa no solo no estuvo visible hasta fines de julio como vaticino nuestro adivino cometario... (Carrasco, 1844).

Posteriormente este imaginario kepleriano-newtoniano se expande a las escuelas de primera enseñanza, así en el año de 1865 ubicamos un libro de educación primaria escrito por Agustín de la Rosa-Toro:

El movimiento de traslación consiste en que la Tierra, al mismo tiempo que está girando al rededor de su eje, da vueltas continuamente al rededor del Sol,... La curva que describe la Tierra en su traslación es una elipse, y en uno de los focos de esta se halla el Sol. (De la Rosa-Toro, 1865: 23).

Sin embargo el imaginario clásico aún no estaba extinto, pues hemos ubicado un libro sobre astronomía publicado en Arequipa nada menos que por un fiscal de la corte superior que aceptaba aún el geocentrismo como una posibilidad en pleno 1857:

La admirable y perpetua vicisitud de la luz y de las tinieblas producida por el movimiento rápido del sol al rededor de la tierra, o por la revolución del Globo terrestre sobre su eje ... determina aquella parte del tiempo que llamamos día natural ó civil... (Sánchez, 1857, p. 305).

\section{CONCLUSIONES}

1. Tres fueron los imaginarios astronómicos presentes en el Perú entre los siglos XVII al XIX: el ptolemaico, el ticónico y el kepleriano-newtoniano.

2. Durante el siglo XVII coexistieron los imaginarios ptolemaico y ticónico.

3. Durante el siglo XVII fue dominante el imaginario ticónico aunque estuvieron presentes también el ptolemaico y el kepleriano.

4. En el siglo XIX fue dominante en imaginario kepleriano-newtoniano 


\section{REFERENCIAS BIBLIOGRÁFICAS}

BUENO, Cosme

1756 El conocimiento de los tiempos, Lima.

CARRASCO, Eduardo

1844 Calendario peruano. Lima.

DELA ROSA-TORO, Agustín

1865 Astronomía. Lima.

RUIZ LOZANO, Francisco

1665 Tratado de cometas, observacion, y ivivio del que se vio en esta civdad de los Reyes, y generalmente en todo el Mundo, por los fines del año de 1664. Y principios defte de 1665. Compuesto por el Capitan Francisco Rviz Lozano Cofmografo mayor defte Reyno, y Cathedratico de Prima de Mathematicas en efta Ciudad, Lima.

\section{GALILEO}

1984 El ensayador. Madrid: Sarpe,

GONZALES GONZALES, Francisco José

1992 Astronomía y navegación en España. Siglos XVI-XVII. Madrid: Mapfre

MORENO, Gabriel

1798 Almanaque peruano y guía de forasteros. Lima.

1805 Almanaque peruano y guía de forasteros. Lima.

NEWTON, Isaac

2011 Principios matemáticos de la filosofía natural. Madrid: Alianza Editorial

SÁNCHEZ, Hipólito

1857 Efemérides astronómicas. Arequipa: Francisco Miranda.

SCHWAB, Federico

1948 Los almanaques peruanos: ¿1680?-1874. Lima, CIP, 1948. Separata del Boletín bibliográfico. Año XXI. Vol. XIX. №s 1-2, Lima. 